\title{
Risk factors for stomach cancer: a systematic review and meta-analysis
}

\author{
Jalal Poorolajal ${ }^{1,2,3}$, Leila Moradi ${ }^{1}$, Younes Mohammadi ${ }^{1,4}$, Zahra Cheraghi ${ }^{1,3}$, \\ Fatemeh Gohari-Ensaf' \\ 'Department of Epidemiology, School of Public Health, Hamadan University of Medical Sciences, Hamadan, Iran; ${ }^{2}$ Research Center for Health \\ Sciences, Hamadan University of Medical Sciences, Hamadan, Iran; ${ }^{3}$ Modeling of Noncommunicable Diseases Research Center, Hamadan \\ University of Medical Sciences, Hamadan, Iran; ${ }^{4}$ Social Determinants of Health Research Center, Hamadan University of Medical Sciences, \\ Hamadan, Iran
}

OBJECTIVES: This report provides information on 14 behavioral and nutritional factors that can be addressed in stomach cancer prevention programs.

METHODS: PubMed, Web of Science, and Scopus were searched through December 2018. Reference lists were also screened. Observational studies addressing the associations between stomach cancer and behavioral factors were analyzed. Between-study heterogeneity was investigated using the $\chi^{2}, \tau^{2}$, and $\mathrm{I}^{2}$ statistics. The likelihood of publication bias was explored using the Begg and Egger tests and trim-and-fill analysis. Effect sizes were expressed as odds ratios (ORs) with 95\% confidence intervals (CIs) using a random-effects model.

RESULTS: Of 52,916 identified studies, 232 (including 33,831,063 participants) were eligible. The OR (95\% CI) of factors associated with stomach cancer were as follows: Helicobacter pylori infection, 2.56 (95\% CI, 2.18 to 3.00); current smoking, 1.61 (95\% CI, 1.49 to 1.75); former smoking 1.43 (95\% CI, 1.29 to 1.59); current drinking, 1.19 (95\% CI, 1.10 to 1.29); former drinking, 1.73 (95\% CI, 1.17 to 2.56); overweight/obesity, 0.89 (95\% CI, 0.74 to 1.08 ); sufficient physical activity, 0.83 (95\% CI, 0.68 to 1.02 ); consumption of fruits $\geq 3$ times/wk, 0.48 (95\% CI, 0.37 to 0.63 ); consumption of vegetables $\geq 3$ times/wk, 0.62 (95\% CI, 0.49 to 0.79); eating pickled vegetables, 1.28 (95\% CI, 1.09 to 1.51); drinking black tea, 1.00 (95\% CI, 0.84 to 1.20 ); drinking green tea, 0.88 (95\% CI, 0.80 to 0.97 ); drinking coffee, 0.99 (95\% CI, 0.88 to 1.11); eating fish $\geq 1$ time/wk 0.79 (95\% CI, 0.61 to 1.03 ); eating red meat $\geq 4$ times/wk 1.31 (95\% CI, 0.87 to 1.96), and high salt intake 3.78 (95\% CI, 1.74 to 5.44 ) and 1.34 (95\% CI, 0.88 to 2.03 ), based on two different studies.

CONCLUSIONS: This meta-analysis provided a clear picture of the behavioral and nutritional factors associated with the development of stomach cancer. These results may be utilized for ranking and prioritizing preventable risk factors to implement effective prevention programs.

KEY WORDS: Stomach neoplasms, Gastric neoplasms, Risk factors, Behavior, Nutrition status, Meta-analysis

Correspondence: Leila Moradi

Department of Epidemiology, School of Public Health, Hamadan University of Medical Sciences, Hamadan 65157838695, Iran E-mail: leilamoradi073@gmail.com

Received: Dec 11, 2019 / Accepted: Feb 2, 2020 / Published: Feb 2, 2020

This article is available from: http://e-epih.org/

(c) This is an open-access article distributed under the terms of the Creative Commons Attribution License (https://creativecommons.org/licenses/by/4.0/), which permits unrestricted use, distribution, and reproduction in any medium, provided the original work is properly cited.

(C) 2020, Korean Society of Epidemiology

\section{INTRODUCTION}

Stomach cancer, also known as gastric cancer, is the fifth most frequent type of cancer and the third-leading cause of cancer-related death worldwide, responsible for over 1,000,000 new cases and an estimated 783,000 deaths in 2018 [1].

Many factors may play a role in the development of stomach cancer. Advanced age [2], male sex [1], ethnicity [3], and genetic factors [4] may contribute to the development of stomach cancer, but they are neither modifiable nor preventable. However, nutri- 
tional factors [5] and behavioral factors such as cigarette smoking [6] and drinking alcohol [6,7], as well as Helicobacter pylori infection [8], also contribute to the development of stomach cancer. These factors are largely modifiable and preventable, and therefore can be considered when designing effective prevention programs.

Efforts to improve screening programs and the early detection and treatment of stomach cancer are important, but taking action to address preventable factors that play a role in the development of stomach cancer is a priority. Ranking and prioritizing the factors that contribute to stomach cancer and implementing prevention programs can prevent thousands of cases of stomach cancer each year. Effective intervention strategies and prevention programs require a comprehensive understanding and a clear picture of the factors that promote stomach cancer. No comprehensive systematic review has yet been conducted to address all the potential behavioral and nutritional factors that play a pivotal role in the development of stomach cancer. This systematic review was conducted to address the associations between stomach cancer and 14 potentially modifiable behavioral and nutritional factors that may be addressed in prevention programs aimed at reducing the incidence of stomach cancer.

\section{MATERIALS AND METHODS}

\section{Eligibility criteria}

The outcome of interest was pathologically confirmed stomach cancer, of any type (adenocarcinoma, lymphoma, sarcoma, or carcinoid) and location (cardia or non-cardia), among the general population, regardless of age, sex, race, ethnicity, and geographical region. The exposures of interest are listed below:

H. pylori infection, regardless of cytotoxin-associated gene A (CagA) pathogenicity (positive vs. negative); Cigarette smoking (current/former smokers vs. non-smokers); Drinking alcohol (current/former drinkers vs. non-drinkers); Body mass index (BMI; overweight/obese vs. normal weight); Physical activity (sufficient vs. insufficient); Fruit consumption ( $\geq 7$ times/wk vs. $<7$ times/wk and $\geq 3$ times/wk vs. $<3$ times/wk); Vegetable consumption $(\geq 7$ times/wk vs. $<7$ times/wk and $\geq 3$ times/wk vs. $<3$ times/wk); Consumption of pickled vegetables (yes vs. no); Drinking black tea (yes vs. no); Drinking green tea (yes vs. no); Drinking coffee (yes vs. no); Fish consumption ( $\geq 1$ serving/wk vs. $<1$ serving/wk); Red meat consumption ( $\geq 4$ times/wk vs. $<4$ times/wk); Salt intake ( $>5 \mathrm{~g} / \mathrm{d}$ vs. $\leq 5 \mathrm{~g} / \mathrm{d}$ ); A BMI of $18.5-24.9 \mathrm{~kg} / \mathrm{m}^{2}$ was classified as normal weight, $25.0-29.9 \mathrm{~kg} / \mathrm{m}^{2}$ as overweight, and $\geq 30.0 \mathrm{~kg} / \mathrm{m}^{2}$ as obese.

At least 60 minutes of moderate- to vigorous-intensity physical activity per day (or $300 \mathrm{~min} / \mathrm{wk}$ ) was considered sufficient for adults [9].

Observational (cohort and case-control) studies addressing the association between stomach cancer and any of the above factors were included in the meta-analysis, irrespective of language, publication date, and the nationality, race, sex, and age of participants.

\section{Information sources and search}

PubMed, Web of Science, and Scopus were searched through
December 2018. The reference lists of the included studies were also explored. The following terms were searched: (stomach cancer OR gastric cancer OR stomach neoplasms OR gastric neoplasms OR gastric malignancy OR stomach malignancy OR stomach tumor OR gastric tumor) AND (Helicobacter pylori OR H. pylori OR smoking OR cigarette OR tobacco products OR tobacco OR alcohol OR ethanol OR body mass index OR BMI OR overweight OR obesity OR obese OR physical activity OR exercise OR fruit OR vegetable OR pickled OR meat OR coffee OR tea OR fish OR salt OR sodium chloride).

\section{Study selection}

The search results of all databases were combined using EndNote, and duplicates were deleted. Then, 2 authors (LM and FG) independently screened the titles and abstracts and excluded ineligible studies. The full texts of potentially relevant studies were retrieved for further evaluation.

\section{Data extraction}

The data from the relevant studies were extracted by 2 authors (LM and JP) using an electronic data collection form prepared in Stata (StataCorp., College Station, TX, USA).

\section{Methodological quality}

The Newcastle-Ottawa Scale (NOS) [10] was used to assess the methodological quality of the included studies. Based on this scale, a maximum of 9 stars was assigned to each study. Studies that received 7 or more stars were labeled high-quality, and otherwise studies were classified as low-quality.

\section{Heterogeneity and publication bias}

The heterogeneity across studies was examined using the chisquare $\left(\chi^{2}\right)$ test [11] and tau-square $\left(\tau^{2}\right)$ test and was quantified by the $\mathrm{I}^{2}$ statistic [12]. According to the $\mathrm{I}^{2}$ value, heterogeneity was classified as low $(<50 \%)$, moderate $(50-74 \%)$, or high $(\geq 75 \%)$.

The possibility of publication bias was explored by the Egger [13] and Begg [14] tests and the trim-and-fill method [15].

\section{Summary measures}

The effect measure of choice was the odds ratio (OR), rate ratio, or hazard ratio with $95 \%$ confidence intervals (CIs). However, we analyzed these effect measures separately.

The results were reported based on a random-effects model [16]. The data were analyzed at a significance level of 0.05 using Stata version 14.2 (StataCorp., College Station, TX, USA) and Review Manager version 5.3 (https://review-manager.software.informer.com/5.3/).

\section{Sensitivity analysis}

If the between-study heterogeneity was moderate to high $\left(\mathrm{I}^{2}\right.$ $\geq 50 \%$ ), the source of heterogeneity was investigated using a sequential algorithm [17]. 


\section{Ethics statement}

This study was a systematic review in which no human subject or animal was employed.

Identification

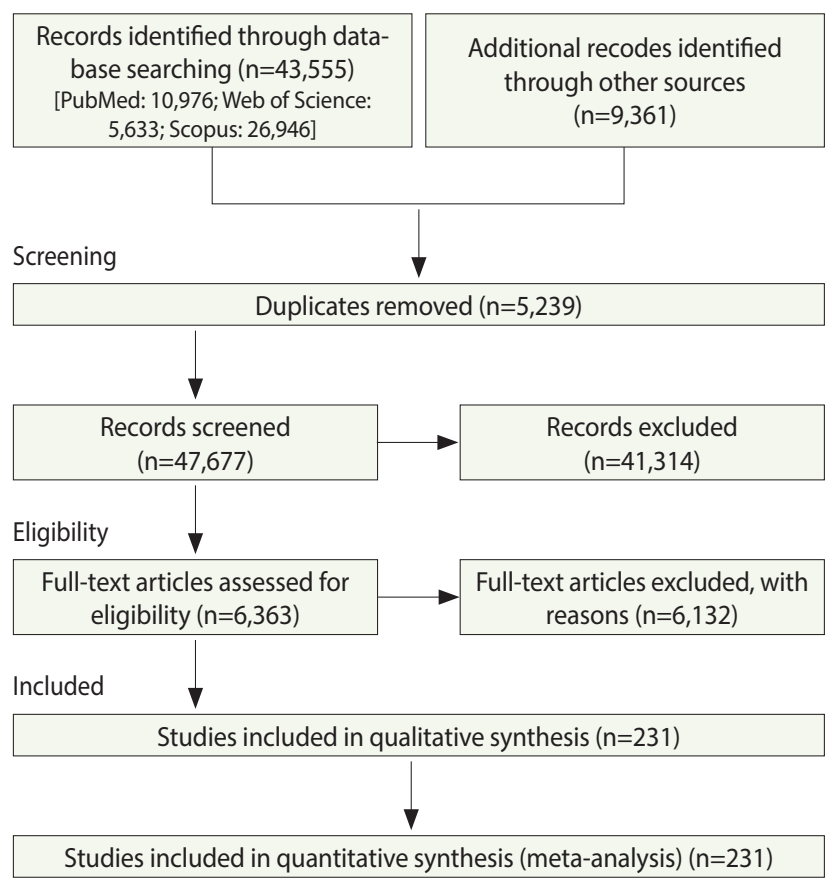

Figure 1. Flow of information through the various phases of the systematic review.

\section{RESULTS}

\section{Description of studies}

In total, 52,916 studies were identified, including 43,555 studies obtained by searching the electronic databases through December 2018 and 9,359 articles identified by searching the reference lists of the included studies. After excluding duplicates and ineligible studies, 232 studies with 33,831,063 participants (Supplementary Material 1) were included in the meta-analysis (Figure 1).

\section{Synthesis of results \\ H. pylori infection}

Based on 68 studies (Supplementary Material 2), the overall OR for positive versus negative $H$. pylori infection status was 2.56 (95\% CI, 2.18 to 3.00). The overall effect measure showed that $H$. pylori infection significantly increased the risk of stomach cancer by more than 2.5 -fold $(\mathrm{p}=0.001)$. Between-study heterogeneity was high $\left(\mathrm{I}^{2}=86 \%\right)$. The overall effect became weaker (OR, 2.13; $95 \% \mathrm{CI}, 1.89$ to $2.41 ; \mathrm{I}^{2}=47 \%$ ) after performing a sensitivity analysis (Table 1).

The Begg test $(\mathrm{p}=0.004)$, but not the Egger test $(\mathrm{p}=0.122)$, revealed evidence of publication bias. Trim-and-fill analysis estimated 4 missing studies (Figure 2). The overall effect measure based on this analysis was an OR of 2.42 (95\% CI, 2.06 to 2.83), which was slightly weaker than the originally reported overall effect measure.

\section{Cigarette smoking}

Based on 77 studies (Supplementary Material 3), the overall OR for current smokers versus never smokers was 1.61 (95\% CI, 1.49

Table 1. Results of the sensitivity analysis

\begin{tabular}{|c|c|c|c|c|c|c|c|c|}
\hline \multirow{3}{*}{ Variables } & \multicolumn{8}{|c|}{ Sensitivity analysis } \\
\hline & \multicolumn{4}{|c|}{ Before } & \multicolumn{4}{|c|}{ After } \\
\hline & Study (n) & $\chi^{2}$ & $\mathrm{I}^{2}(\%)$ & OR $(95 \% \mathrm{Cl})$ & Study (n) & $\chi^{2}$ & $\mathrm{I}^{2}(\%)$ & OR $(95 \% \mathrm{Cl})$ \\
\hline Helicobacter pylori & 68 & 0.001 & 86 & $2.56(2.18,3.00)$ & 49 & 0.003 & 47 & $2.13(1.89,2.41)$ \\
\hline \multicolumn{9}{|l|}{ Smoking } \\
\hline Current & 95 & 0.001 & 78 & $1.61(1.49,1.75)$ & 73 & 0.001 & 49 & $1.66(1.54,1.79)$ \\
\hline Former & 52 & 0.001 & 65 & $1.43(1.29,1.59)$ & 50 & 0.001 & 44 & $1.35(1.24,1.47)$ \\
\hline \multicolumn{9}{|l|}{ Alcohol } \\
\hline Current & 72 & 0.001 & 83 & $1.19(1.10,1.29)$ & 60 & 0.001 & 50 & $1.05(0.99,1.11)$ \\
\hline Former & 11 & 0.001 & 84 & $1.73(1.17,2.56)$ & 9 & 0.050 & 48 & $2.01(1.48,2.72)$ \\
\hline Body mass index & 14 & 0.001 & 86 & $0.89(0.74,1.08)$ & 10 & 0.090 & 41 & $1.14(1.03,1.26)$ \\
\hline Physical activity & 7 & 0.090 & 45 & $0.83(0.68,1.02)$ & NA & - & - & - \\
\hline Fruit consumption & 14 & 0.001 & 86 & $0.48(0.37,0.63)$ & 11 & 0.070 & 42 & $0.64(0.55,0.75)$ \\
\hline Vegetable consumption & 9 & 0.001 & 74 & $0.62(0.49 .0 .79)$ & 6 & 0.140 & 40 & $0.70(0.58,0.84)$ \\
\hline Pickled vegetable consumption & 16 & 0.060 & 39 & $1.28(1.09,1.51)$ & NA & - & - & - \\
\hline Black tea intake & 13 & 0.002 & 62 & $1.00(0.84,1.20)$ & 12 & 0.120 & 34 & $0.94(0.83,1.07)$ \\
\hline Green tea intake & 16 & 0.220 & 22 & $0.88(0.80,0.97)$ & NA & - & - & - \\
\hline Coffee intake & 12 & 0.160 & 29 & $0.99(0.88,1.11)$ & NA & - & - & - \\
\hline Fish consumption & 11 & 0.001 & 76 & $0.79(0.61,1.03)$ & 9 & 0.070 & 45 & $0.68(0.55,0.83)$ \\
\hline Red meat consumption & 7 & 0.001 & 83 & $1.31(0.87,1.96)$ & 4 & 0.340 & 11 & $0.91(0.77,1.09)$ \\
\hline
\end{tabular}

$\mathrm{OR}$, odds ratio; $\mathrm{Cl}$, confidence interval; $\mathrm{NA}$, not available. 


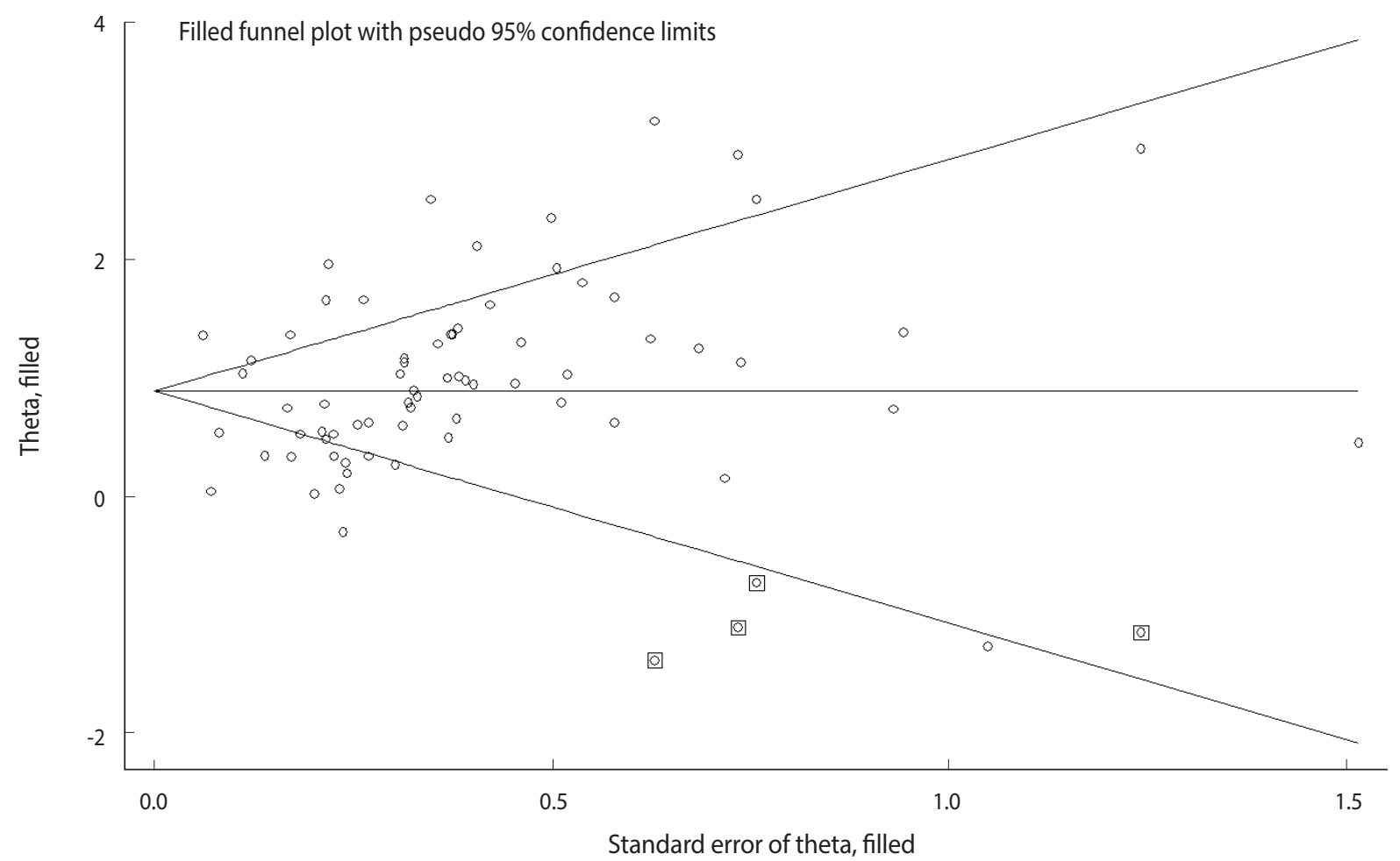

Figure 2. Trim-and-fill analysis estimating the number of possible missing studies for the association between stomach cancer and Helicobacter pylori infection. The squares represent the possible missing studies.

to 1.75). The overall effect measure showed that current smoking significantly increased the risk of stomach cancer by $61 \%(\mathrm{p}=0.001)$. Between-study heterogeneity was high $\left(\mathrm{I}^{2}=78 \%\right)$. The overall effect became slightly stronger (OR, 1.66; 95\% CI, 1.54 to $1.79 ; \mathrm{I}^{2}=49 \%$ ) after performing a sensitivity analysis (Table 1 ).

In addition, based on 66 studies (Supplemental Material 4), the overall OR for former smokers versus never smokers was 1.43 (95\% CI, 1.29 to 1.59 ). The overall effect measure showed that former smoking significantly increased the risk of stomach cancer by $43 \%$ $(\mathrm{p}=0.001)$. Between-study heterogeneity was moderate $\left(\mathrm{I}^{2}=65 \%\right)$. The overall effect became slightly weaker (OR, 1.35; 95\% CI, 1.24 to $1.47 ; \mathrm{I}^{2}=44 \%$ ) after performing a sensitivity analysis (Table 1 ).

The Begg test revealed no evidence of publication bias $(\mathrm{p}=0.722)$, but the Egger test did show evidence of publication bias $(\mathrm{p}=0.001)$. Trim-and-fill analysis estimated 19 missing studies, but the overall effect measure did not change significantly.

\section{Drinking alcohol}

Based on 84 studies (Supplementary Material 5), the overall OR for current drinkers versus never drinkers was 1.19 (95\% CI, $1.10,1.29)$. The overall effect measure showed that current drinking significantly increased the risk of stomach cancer by $19 \%$ ( $\mathrm{p}=$ $0.001)$. Between-study heterogeneity was high $\left(\mathrm{I}^{2}=83 \%\right)$. The overall effect became slightly weaker (OR, 1.05; 95\% CI, 0.99 to $1.11 ; \mathrm{I}^{2}=50 \%$ ) after performing a sensitivity analysis (Table 1 ).

In addition, based on 16 studies (Supplementary Material 6), the overall OR for former drinking versus never drinking was 1.73 (95\% CI, 1.17 to 2.56). The overall effect measure showed that former drinking significantly increased the risk of stomach cancer by $73 \%(\mathrm{p}=0.004)$. Between-study heterogeneity was high $\left(\mathrm{I}^{2}=\right.$ $84 \%$ ). The overall effect became slightly weaker (OR, 2.01; 95\% CI, 1.48 to $2.72 ; \mathrm{I}^{2}=48 \%$ ) after performing a sensitivity analysis (Table 1). There was no evidence of publication bias.

\section{Body mass index}

Based on 25 studies (Supplementary Material 7), the overall OR for overweight/obesity versus normal weight was 0.89 (95\% CI, 0.74 to 1.08). The overall effect measure showed that overweight/ obesity had no significant effect on stomach cancer $(\mathrm{p}=0.240)$. Between-study heterogeneity was high $\left(\mathrm{I}^{2}=86 \%\right)$. The overall effect changed slightly (OR, 1.14; 95\% CI, 1.03 to 1.26 ; $\left.\mathrm{I}^{2}=41 \%\right)$ after performing a sensitivity analysis (Table 1 ). There was no evidence of publication bias.

\section{Sufficient physical activity}

Based on 11 studies (Supplementary Material 8), the overall OR for sufficient versus insufficient physical activity was 0.83 (95\% CI, 0.68 to 1.02). The overall effect measure showed that physical activity had no significant effect on stomach cancer $(p=0.080)$, which seems negligible. Between-study heterogeneity was low $\left(\mathrm{I}^{2}=45 \%\right)$. There was no evidence of publication bias. 


\section{Fruits}

Based on 13 studies (Supplementary Material 9), the overall OR for fruit consumption $\geq 3$ times/wk versus fruit consumption $<3$ times/wk was 0.48 (95\% CI, 0.37 to 0.63 ). The overall effect measure showed that fruit consumption significantly reduced the risk of stomach cancer by $48 \%(\mathrm{p}=0.001)$. Between-study heterogeneity was high $\left(\mathrm{I}^{2}=86 \%\right)$. The overall effect became slightly weaker (OR, $0.64 ; 95 \%$ CI, 0.55 to $0.75 ; \mathrm{I}^{2}=42 \%$ ) after performing a sensitivity analysis (Table 1$)$. Both the Begg test $(p=0.010)$ and the Egger test $(p=0.001)$ revealed evidence of publication bias, but trim-and-fill analysis did not change the results.

\section{Vegetables}

Based on 18 studies (Supplementary Material 10), the OR for vegetable consumption $\geq 3$ times/wk versus vegetable consumption $<3$ times/wk was 0.62 (95\% CI, 0.49 to 0.79$)$. The overall effect measure showed that vegetable consumption significantly reduced the risk of stomach cancer by $62 \%(\mathrm{p}=0.001)$. Between-study heterogeneity was high $\left(\mathrm{I}^{2}=74 \%\right)$. The overall effect became slightly weaker (OR, $0.70 ; 95 \% \mathrm{CI}, 0.58$ to $0.84 ; \mathrm{I}^{2}=40 \%$ ) after performing a sensitivity analysis (Table 1). There was no evidence of publication bias.

\section{Pickled vegetable}

Based on 19 studies (Supplementary Material 11), the overall OR for consuming versus not consuming pickled vegetables was
1.28 (95\% CI, 1.09 to 1.51$)$. The overall effect measure showed that consuming pickled vegetables significantly increased the risk of stomach cancer by $28 \%(\mathrm{p}=0.001)$. Between-study heterogeneity was low $\left(\mathrm{I}^{2}=39 \%\right)$. There was no evidence of publication bias.

\section{Black tea}

Based on 15 studies (Supplementary Material 12), the overall OR for drinking versus not drinking black tea was 1.00 (95\% CI, 0.84 to 1.20 ). The overall effect measure showed that drinking black tea had no significant effect on stomach cancer $(\mathrm{p}=0.970)$. Between-study heterogeneity was moderate $\left(\mathrm{I}^{2}=62 \%\right)$. The overall effect became slightly stronger (OR, $0.94 ; 95 \%$ CI, 0.83 to 1.07 ; $\mathrm{I}^{2}=34 \%$ ) after performing a sensitivity analysis (Table 1 ). No evidence of publication bias was revealed.

\section{Green tea}

Based on 16 studies (Supplementary Material 13), the overall OR for drinking versus not drinking green tea was 0.88 (95\% CI, 0.80 to 0.97$)$. The overall effect measure showed that drinking green tea had no significant effect on stomach cancer $(\mathrm{p}=0.010)$. Between-study heterogeneity was low $\left(\mathrm{I}^{2}=22 \%\right)$. No evidence of publication bias was seen.

\section{Coffee}

Based on 14 studies (Supplementary Material 14), the overall

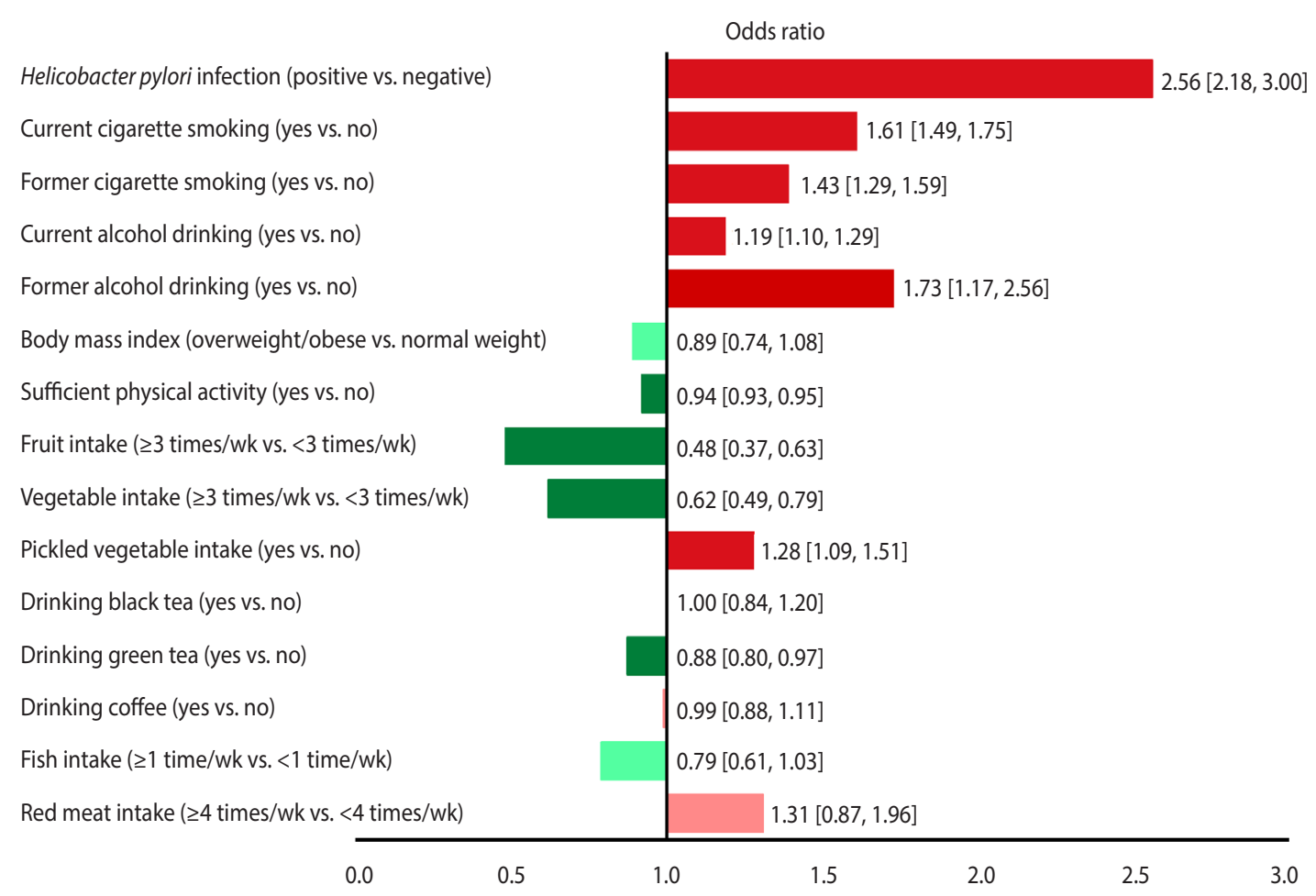

Figure 3. The associations (95\% confidence intervals) between stomach cancer and nutritional and behavioral factors in a single view. Protective factors are shown in green (dark green, significant; light green, non-significant) and risk factors are shown in red (dark red, significant; light red, non-significant). 
OR for drinking coffee versus not drinking coffee was 0.99 (95\% CI, 0.88 to 1.11). The overall effect measure showed that coffee drinking had no significant effect on stomach cancer $(p=0.820)$. Between-study heterogeneity was moderate $\left(\mathrm{I}^{2}=29 \%\right)$. There was no evidence of publication bias.

\section{Fish}

Based on 11 studies (Supplementary Material 15), the OR for eating fish $\geq 1$ time/wk versus $<1$ time/wk was 0.79 (95\% CI, 0.61 to 1.03). The overall effect showed that fish consumption had no significant effect on stomach cancer $(p=0.080)$. Between-study heterogeneity was high $\left(\mathrm{I}^{2}=76 \%\right)$. The overall effect became stronger (OR, 0.68 ; 95\% CI, 0.55 to $0.83 ; \mathrm{I}^{2}=45 \%$ ) after performing a sensitivity analysis (Table 1). No evidence of publication bias was seen.

\section{Red meat}

Based on 11 studies (Supplementary Material 16), the overall OR for eating red meat $\geq 4$ times/wk versus $<4$ times/wk was 1.31 (95\% CI, 0.87 to 1.96). The overall effect measure showed that consumption of red meat had no significant effect on stomach cancer $(\mathrm{p}=0.080)$. Between-study heterogeneity was high $\left(\mathrm{I}^{2}=83 \%\right)$. The overall effect changed slightly (OR, $0.91 ; 95 \% \mathrm{CI}, 0.77$ to $1.09 ; \mathrm{I}^{2}=$ $11 \%$ ) after performing a sensitivity analysis (Table 1 ). There was no evidence of publication bias.

\section{Salt}

Only 2 studies addressed the association between high salt intake and stomach cancer. The results of the two studies are reported separately rather than a pooled OR because of the number of studies was limited. According to these studies, the OR for salt intake of $>5 \mathrm{~g} / \mathrm{d}$ versus $\leq 5 \mathrm{~g} / \mathrm{d}$ was 3.78 (95\% CI, 1.74 to 5.44 ) [18] and 1.34 (95\% CI, 0.88 to 2.03) [19], respectively. Both studies reported that a high intake of salt significantly increased the risk of stomach cancer.

Figure 3 presents a unified overview of the associations between stomach cancer and all nutritional and behavioral factors. As shown in this figure, $H$. pylori infection, current and former cigarette smoking, current and former alcohol drinking, and pickled vegetable consumption were found to significantly increase the risk of stomach cancer. In contrast, sufficient physical activity, fruit consumption, and vegetable consumption significantly reduced the risk of stomach cancer. Meanwhile, BMI, drinking black tea, green tea, and coffee, and eating fish and red meat had no statistically significant effects on the risk of stomach cancer.

\section{DISCUSSION}

According to our findings, $H$. pylori infection and smoking were the first and second most powerful risk factors for stomach cancer, respectively, whereas fruit and vegetable consumption were the first and second most powerful protective factors against stomach cancer, respectively.

The magnitudes of the measures of association reported in this systematic review may be used for ranking and prioritizing the relative importance of risk and protective factors. However, it should be kept in mind that these factors vary in terms of their physiological modus operandi and their units of exposure. Therefore, direct comparisons are often unwarranted [20]. In other words, the mere fact that the ORs of some risk factors for stomach cancer are higher than the ORs of other risk factors is not a sufficient basis for ranking and prioritizing risk factors. Instead, the prevalence of risk factors in the community is an essential criterion that must be taken into account when ranking and prioritizing risk factors. When the association between a particular risk factor and the outcome of interest is strong (a high OR), but the prevalence of that risk factor is low in the community, the overall impact of the risk factor on the disease burden in the community is low. In contrast, when a particular risk factor is common in the community, the overall impact of the factor on the outcome of interest may be tremendous even if the association between the risk factor and the outcome is not as strong (a low OR). Therefore, ranking and prioritizing the behavioral and nutritional factors affecting stomach cancer risk depends on both the strength of the associations (the magnitude of ORs) and the prevalence of the factors in the community.

Our results indicated that $H$. pylori infection was strongly associated with the development of stomach cancer. Based on the available evidence, $H$. pylori infection induces stomach cancer through direct and indirect pathways. The direct action of $H$. pylori on gastric epithelial cells is thought to be mediated by the induction of protein modulation and genetic mutations. Its indirect action on gastric epithelial cells is thought to be through inflammation. Both pathways work together to promote gastric carcinogenesis [21]. In addition, CagA apparently interacts with some host proteins that regulate cell growth, cell motility, and cell polarity. These interactions with CagA induce morphological transformations that may predispose cells to epigenetic changes involved in gastric carcinogenesis [22].

Our results revealed a positive relationship between cigarette smoking and the development of stomach cancer. Cigarette smoke contains over 7,000 toxic chemicals, including human carcinogens [23]. These toxins and carcinogens can cause direct DNA damage. Since DNA controls cells' normal growth and function, DNA damage can alter cells' growth patterns, and abnormal gastric epithelial cells with DNA damage can turn into cancer $[24,25]$.

This systematic review showed that drinking alcohol increased the risk of developing stomach cancer. Acetaldehyde, the first and most toxic metabolite of ethanol, is a human carcinogen that can induce DNA lesions by inhibiting DNA methylation and by interacting with retinoid metabolism [26]. DNA lesions may lead to cell mutations, which convert a normal cell into cancer [27]. In addition, alcohol can act as an irritant and cause mucosal damage. The damaged cells may try to repair themselves, which could lead to DNA changes that can be a step toward cancer [28].

According to our results, the risk of stomach cancer of former drinkers was higher than that of current drinkers. One possible 
explanation for this finding is that former drinkers might be heavy drinkers who had drunk alcohol for many years, but were forced to quit drinking alcohol because of severe liver and gastric complications.

Pickled vegetables may increase the risk of stomach cancer because they contain large amounts of salt and because key nutrients are lost in vegetables under acidic and oxygenic conditions $[29,30]$. Furthermore, pickled vegetables are considered to be a possible source of nitrosamines, which may contribute to gastric carcinogenesis. The contamination of pickled vegetables with fungi has also been postulated to contribute to the incidence of stomach cancer [31].

Based on our findings, fruit and vegetable consumption was associated with a substantial reduction in stomach cancer risk. It has been postulated that the anti-carcinogenic effects of fruits and vegetables may be attributed to the antioxidant effect of their vitamin content, especially vitamin $\mathrm{C}$ and beta-carotene. Antioxidants neutralize reactive oxygen free radicals, which cause DNA damage $[32,33]$. Damaged DNA may lead to genetic modifications and carcinogenesis [24,25].

Our results showed a protective, but non-significant accusation between stomach cancer and overweight and obesity. However, the between-study heterogeneity was high $\left(\mathrm{I}^{2}=86 \%\right)$. When we performed a sensitivity analysis, the overall effect changed from protective to a significant risk elevation (OR, 1.14; 95\% CI, 1.03 to 1.26). Chen et al. [34]. conducted a meta-analysis including studies published before 2013 that were indexed in MEDLINE and EMBASE to address the association between gastric cancer and BMI. They reported that the relative risk of gastric cancer was 1.01 (95\% CI, 0.96 to 1.07) for overweight and 1.06 (95\% CI, 0.99 to 1.12) for obesity, and neither of those associations was statistically significant. Based on the current evidence, BMI does not seem to have a significant effect on the incidence of stomach cancer.

This systematic review has a few limitations and potential biases. There were some studies, mostly old, that seemed potentially eligible to be included in this meta-analysis, but neither their full texts nor their corresponding authors were accessible. This issue might have introduced selection bias in our results. Furthermore, several epidemiological studies that investigated the associations between stomach cancer and some nutritional and behavioral risk factors were excluded from the meta-analysis because they were not consistent with the inclusion criteria of this review. This issue may also raise the possibility of selection bias.

\section{CONCLUSION}

This meta-analysis provided a clear picture of several behavioral and nutritional factors that play pivotal roles in the development of stomach cancer. These results are helpful and may be utilized for ranking and prioritizing preventable risk factors to implement effective interventions and community-based prevention programs. We reemphasize that both the strength of associations and the prevalence of factors in the community should be taken into account when ranking and prioritizing stomach cancer-associated factors.

\section{SUPPLEMENTARY MATERIALS}

Supplementary materials are available at http://www.e-epih.org/.

\section{CONFLICT OF INTEREST}

The authors have no conflicts of interest to declare for this study.

\section{FUNDING}

The Vice-Chancellor of Research and Technology, Hamadan University of Medical Sciences funded this study (No. 9610266919).

\section{ACKNOWLEDGEMENTS}

These results were obtained as part of an MSc thesis in Epidemiology. We would like to appreciate the Vice-Chancellor for Research and Technology of the Hamadan University of Medical Sciences for approval and financial support of this study.

\section{AUTHOR CONTRIBUTIONS}

Conceptualization: JP. Data curation: JP, LM, YM, ZC. Formal analysis: JP, LM. Funding acquisition: JP. Methodology: JP, LM, FGE. Writing - original draft: JP, ZC, YM. Writing - review \& editing: LM, YM, ZC, FGE.

\section{ORCID}

Jalal Poorolajal: https://orcid.org/0000-0002-3758-3006; Leila Moradi: https://orcid.org/0000-0003-3145-9546; Younes Mohammadi: https://orcid.org/0000-0001-7521-4909; Zahra Cheraghi: https://orcid.org/0000-0001-9041-559X; Fatemeh Gohari-Ensaf: https://orcid.org/0000-0001-9369-1549

\section{REFERENCES}

1. Bray F, Ferlay J, Soerjomataram I, Siegel RL, Torre LA, Jemal A. Global cancer statistics 2018: GLOBOCAN estimates of incidence and mortality worldwide for 36 cancers in 185 countries. CA Cancer J Clin 2018;68:394-424.

2. Hisamichi S, Sasaki R, Sugawara N, Yanbo T, Yamagata S. Stomach cancer in various age groups (Japan) as detected by gastric mass survey. J Am Geriatr Soc 1979;27:439-443.

3. Dockerty JD, Marshall S, Fraser J, Pearce N. Stomach cancer in New Zealand: time trends, ethnic group differences and a cancer registry-based case-control study. Int J Epidemiol 1991;20:45-53.

4. Sun H, Wu X, Wu F, Li Y, Yu Z, Chen X, et al. Associations of genetic variants in the PSCA, MUC1 and PLCE1 genes with stomach cancer susceptibility in a Chinese population. PLoS One 2015; 


\section{0:e0117576.}

5. Mathew A, Gangadharan P, Varghese C, Nair MK. Diet and stomach cancer: a case-control study in South India. Eur J Cancer Prev 2000;9:89-97.

6. Praud D, Rota M, Pelucchi C, Bertuccio P, Rosso T, Galeone C, et al. Cigarette smoking and gastric cancer in the Stomach Cancer Pooling (StoP) Project. Eur J Cancer Prev 2018;27:124-133.

7. Minami Y, Kanemura S, Oikawa T, Suzuki S, Hasegawa Y, Miura $\mathrm{K}$, et al. Associations of cigarette smoking and alcohol drinking with stomach cancer survival: a prospective patient cohort study in Japan. Int J Cancer 2018;143:1072-1085.

8. González CA, Sala N, Rokkas T. Gastric cancer: epidemiologic aspects. Helicobacter 2013;18 Suppl 1:34-38.

9. World Health Organization. Physical activity and young people; 2018 [cited 2018 Nov 12]. Available from: https://www.who.int/ dietphysicalactivity/factsheet_young_people/en/.

10. Wells G, Shea B, O'connell DL, Peterson J, Welch V, Losos M, et al. The Newcastle-Ottawa Scale (NOS) for assessing the quality of nonrandomised studies in meta-analyses; 2018 [cited $2020 \mathrm{Feb}$ 10]. Available from: http://www.ohri.ca/programs/clinical_epidemiology/oxford.asp.

11. Higgins JP, Green S. Cochrane handbook for systematic reviews of interventions; 2008 [cited 2020 Feb 10]. Available from: https://www.radioterapiaitalia.it/wp-content/uploads/2017/01/cochrane-handbook-for-systematic-reviews-of-interventions.pdf.

12. Higgins JP, Thompson SG, Deeks JJ, Altman DG. Measuring inconsistency in meta-analyses. BMJ 2003;327:557-560.

13. Egger M, Davey Smith G, Schneider M, Minder C. Bias in metaanalysis detected by a simple, graphical test. BMJ 1997;315:629634.

14. Begg CB, Mazumdar M. Operating characteristics of a rank correlation test for publication bias. Biometrics 1994;50:1088-1101.

15. Duval S, Tweedie R. A nonparametric "trim and fill" method of accounting for publication bias in meta-analysis. J Am Stat Assoc 2000;95:89-98.

16. DerSimonian R, Laird N. Meta-analysis in clinical trials. Control Clin Trials 1986;7:177-188.

17. Patsopoulos NA, Evangelou E, Ioannidis JP. Sensitivity of betweenstudy heterogeneity in meta-analysis: proposed metrics and empirical evaluation. Int J Epidemiol 2008;37:1148-1157.

18. Zhang Z, Zhang X. Salt taste preference, sodium intake and gastric cancer in China. Asian Pac J Cancer Prev 2011;12:1207-1210.

19. Zhong C, Li KN, Bi JW, Wang BC. Sodium intake, salt taste and gastric cancer risk according to Helicobacter pylori infection, smoking, histological type and tumor site in China. Asian Pac J Cancer Prev 2012;13:2481-2484.
20. Szklo M, Nieto FJ. Epidemiology: beyond the basics. 4th ed. Burlington: Jones \& Bartlett Learning; 2019, p. 115.

21. Chiba T, Marusawa H, Seno H, Watanabe N. Mechanism for gastric cancer development by Helicobacter pylori infection. J Gastroenterol Hepatol 2008;23:1175-1181.

22. Hatakeyama M, Higashi H. Helicobacter pylori CagA: a new paradigm for bacterial carcinogenesis. Cancer Sci 2005;96:835-843.

23. World Health Organization. World No Tobacco Day 2017: beating tobacco for health, prosperity, the environment and national development; 2017 [cited 2017 Jun 1]. Available from: http:// www.who.int/mediacentre/news/releases/2017/no-tobacco-day/ en/.

24. Dyke GW, Craven JL, Hall R, Garner RC. Smoking-related DNA adducts in human gastric cancers. Int J Cancer 1992;52:847-850.

25. Pfeifer GP, Denissenko MF, Olivier M, Tretyakova N, Hecht SS, Hainaut P. Tobacco smoke carcinogens, DNA damage and p53 mutations in smoking-associated cancers. Oncogene 2002;21: 7435-7451.

26. Seitz HK, Stickel F. Molecular mechanisms of alcohol-mediated carcinogenesis. Nat Rev Cancer 2007;7:599-612.

27. Deman J, Van Larebeke N. Carcinogenesis: mutations and mutagens. Tumour Biol 2001;22:191-202.

28. Huh K, Kwon TH, Shin US, Kim WB, Ahn BO, Oh TY, et al. Inhibitory effects of DA-9601 on ethanol-induced gastrohemorrhagic lesions and gastric xanthine oxidase activity in rats. J Ethnopharmacol 2003;88:269-273.

29. Kim HJ, Lim SY, Lee JS, Park S, Shin A, Choi BY, et al. Fresh and pickled vegetable consumption and gastric cancer in Japanese and Korean populations: a meta-analysis of observational studies. Cancer Sci 2010;101:508-516.

30. Yalim S, Ozdemir Y. Effects of preparation procedures on ascorbic acid retention in pickled hot peppers. Int J Food Sci Nutr 2003; 54:291-296.

31. Yang CS. Research on esophageal cancer in China: a review. Cancer Res 1980;40:2633-2644.

32. Akyön Y. Effect of antioxidants on the immune response of Helicobacter pylori. Clin Microbiol Infect 2002;8:438-441.

33. Drake IM, Davies MJ, Mapstone NP, Dixon MF, Schorah CJ, White $\mathrm{KL}$, et al. Ascorbic acid may protect against human gastric cancer by scavenging mucosal oxygen radicals. Carcinogenesis 1996;17: 559-562.

34. Chen Y, Liu L, Wang X, Wang J, Yan Z, Cheng J, et al. Body mass index and risk of gastric cancer: a meta-analysis of a population with more than ten million from 24 prospective studies. Cancer Epidemiol Biomarkers Prev 2013;22:1395-1408. 